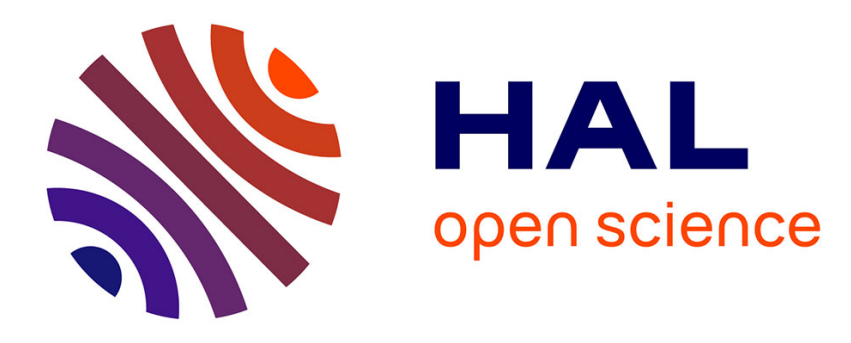

\title{
Tactile objects based on an amplitude disturbed diffraction pattern method
}

Yuan Liu, Jean-Pierre Nikolovski, Nazih Mechbal, Moustapha Hafez, Michel Vergé

\section{- To cite this version:}

Yuan Liu, Jean-Pierre Nikolovski, Nazih Mechbal, Moustapha Hafez, Michel Vergé. Tactile objects based on an amplitude disturbed diffraction pattern method. Applied Physics Letters, 2009, 95 (25), 10.1063/1.3276549 . hal-00749094

\section{HAL Id: hal-00749094 https://hal.science/hal-00749094}

Submitted on 6 Nov 2012

HAL is a multi-disciplinary open access archive for the deposit and dissemination of scientific research documents, whether they are published or not. The documents may come from teaching and research institutions in France or abroad, or from public or private research centers.
L'archive ouverte pluridisciplinaire HAL, est destinée au dépôt et à la diffusion de documents scientifiques de niveau recherche, publiés ou non, émanant des établissements d'enseignement et de recherche français ou étrangers, des laboratoires publics ou privés. 


\title{
Tactile objects based on an amplitude disturbed diffraction pattern method
}

\author{
Yuan Liu, ${ }^{1,2}$ Jean-Pierre Nikolovski, ${ }^{2, a)}$ Nazih Mechbal, ${ }^{1}$ Moustapha Hafez, ${ }^{2}$ and \\ Michel Vergé ${ }^{1}$ \\ ${ }^{1}$ LMSP (CNRS 8106), Arts et Métiers ParisTech, 151 Boulevard de l'Hôpital-75013 Paris, France \\ ${ }^{2}$ CEA, LIST, Sensory Interfaces Laboratory, 18 Route du Panorama, 92265 Fontenay-aux-Roses, France
}

(Received 17 September 2009; accepted 25 November 2009; published online 23 December 2009)

\begin{abstract}
Tactile sensing is becoming widely used in human-computer interfaces. Recent advances in acoustic approaches demonstrated the possibilities to transform ordinary solid objects into interactive interfaces. This letter proposes a static finger contact localization process using an amplitude disturbed diffraction pattern method. The localization method is based on the following physical phenomenon: a finger contact modifies the energy distribution of acoustic wave in a solid; these variations depend on the wave frequency and the contact position. The presented method first consists of exciting the object with an acoustic signal with plural frequency components. In a second step, a measured acoustic signal is compared with prerecorded values to deduce the contact position. This position is then used for human-machine interaction (e.g., finger tracking on computer screen). The selection of excitation signals is discussed and a frequency choice criterion based on contrast value is proposed. Tests on a sandwich plate (liquid crystal display screen) prove the simplicity and easiness to apply the process in various solids. (C) 2009 American Institute of Physics.
\end{abstract}

[doi:10.1063/1.3276549]

In their work, Ing et al. ${ }^{1,2}$ presented an acoustic timereversal process for in solid localization of finger "impact." Comparing with other acoustic approaches such as Rayleigh wave approach ${ }^{3,4}$ or time-of-flight techniques, ${ }^{5,6}$ the timereversal technique can be applied to most solids without any prior knowledge of acoustic properties. However, many human-machine interfaces prefer "touch" sensing than impact localization, because touch is perceived as a more userfriendly interaction. ${ }^{7,8}$ Indeed, some modern interaction modes such as "multitouch" are difficult to achieve with "impacts," since it requires generating several impacts simultaneously on the tactile surface.

Recently, an alternative method based on the crosscorrelation of selective absorption of the vibration modes has been presented. ${ }^{10}$ This process is suitable for static contact localization. However, this process based on an absorption technique cannot address some particular uses such as stylus tip localization. In that case, contact creates more diffraction effect of the propagating acoustic waves than absorption. In this letter, we propose a tactile process based on an amplitude disturbed diffraction pattern (ADDP) phenomenon of an impulse acoustic wave. It expresses the following physical phenomenon: a human finger (or stylus) in contact with the objects affects the acoustic wave propagation in the solid. These disturbances depend on the finger position and the frequency of excited signal. With analysis and a calibration procedure of this variation, we can locate precisely the contact position.

In the following examples, two emitters (piezoelectric transducers Pz27 type by Ferroperm, Denmark) transmit repetitive Lamb waves (period $1 \mathrm{~ms}$ ) in the solid Fig. 1. The excitation signals come from a Tektronix 3012 function generator and are composed of 40 frequency components. These frequencies are uniformly distributed between 20 and 100

\footnotetext{
${ }^{\text {a) }}$ Author to whom correspondence should be addressed. Electronic mail: jean-pierre.nikolovski@cea.fr.
}

$\mathrm{kHz}$ with an interval of $2 \mathrm{kHz}$. When a human finger comes in contact with the object, after a short stabilization time, say less than $1 \mathrm{~ms}$, the acoustic waves are measured with one or two piezoelectric receivers. Typically, acoustic signals are digitized with a Pico ADC 212/50 data acquisition card during a $4 \mathrm{~ms}$ time window at a sampling frequency of 195.31 $\mathrm{kHz}$. A computer is used to drive the function generator and the data acquisition card. Signal processing and image rendering are carried out in the Matlab environment.

Analytically, the received acoustic signal $r(t)$ can be expressed as a function of emitted signal $e(t)$, the object property and geometry $G$, the contact position $P$, and contact surface $S$ at a given pressure

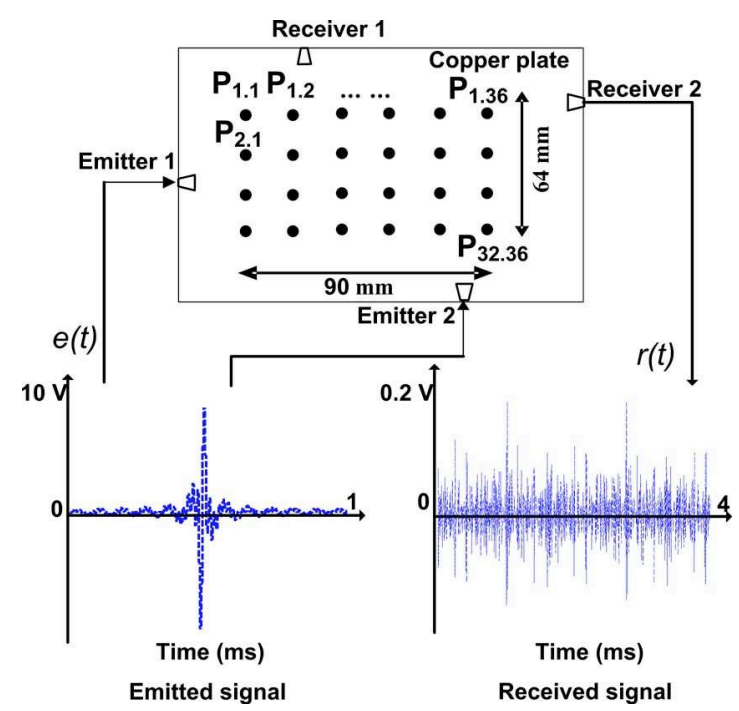

FIG. 1. (Color online) Training step of the disturbed acoustic diffraction tactile process on a copper plate. $32 \times 36$ predefined positions are sequentially touched by an index; received reference signals from two PZT transducers are stored according to the contact positions. 


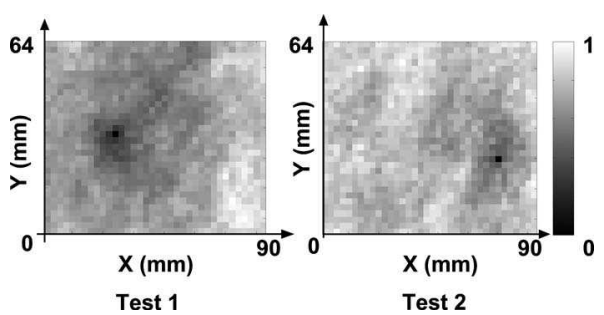

FIG. 2. Contrast images based on the normalized distances from one reference signal to all others. The contact position appears in black. Neighboring points exhibit small distances which implies a limit to the spatial resolution of the learning process.

$$
r(t)=F[e(t), G, P, S] .
$$

The first step is a training step. The tactile surface of the object is divided into an array of predefined "contact points." For a copper plate $100 \times 75 \times 0.45 \mathrm{~mm}^{3}$ large, $36 \times 32$ predefined points are sequentially touched by an artificial silicone finger. The contact surface, roughly $25 \mathrm{~mm}^{2}$, is assured by same contact pressure. The Fourier transform of received signal at each contact point $P_{i, j}$ can be expressed by Eq. (2)

$$
R_{i, j}(f)=F\left(E(f), G, P_{i, j}, S\right) .
$$

In the following example, the $E(f)$ and $G$ are constants, as we maintain the same conditions during the training step. The variation of $S$ is negligible, so we consider that the received signal $r(t)$ is only function of contact position $P$

$$
R_{i, j}(f)=F\left(P_{i, j}, f\right) .
$$

In the second step, the user touches the copper plate, say at position $P^{\prime}$. The measured acoustic signal $r^{\prime}(t)$ is then compared with all stored training signals and an array of distances is calculated

$$
D_{i, j}=\sum_{f}\left|R^{\prime}(f)-R_{i, j}(f)\right| .
$$

The contact position can be visualized on a figure representing the normalized distances with a gray amplitude scale, as in Fig. 2. By using a recognition process of one measured signal among all the reference signals, we are able to find the position of contact.

Compared to the acoustic time-reversal process in which the acoustic signal is produced from the finger impact, meaning the acoustic plate is passive, this method enhances an active acoustic plate featuring a process that continuously generates Lamb wave packets, 1000/s. This tactile technology is said "active" as regards Lamb wave generation, meaning excitation signals are fundamental to its reliability.

We define a disturbed diffracted pattern contrast value as criterion of frequency choice. For that matter which defines another step in the tactile process, the plate is excited with a sinusoidal signal and followed by $70 \times 60$ sequentially contacts on the surface with an interval of $1 \mathrm{~mm}$. The recorded signals are compared with the original signal obtained without contact. Figure 3 shows disturbed diffraction patterns at two different frequencies, one at $60 \mathrm{kHz}$, nonresonant, the other at $79.620 \mathrm{kHz}$, resonant. Images reveal different contrast features which is quantified with the contrast value formula.

Beforehand we calculate with Eq. (5) the mean derivative values of ADDP in the neighborhood of each contact
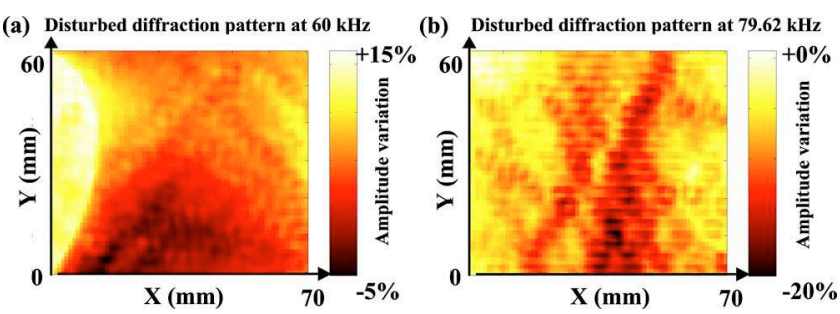

FIG. 3. (Color online) ADDP based on relative amplitude variations according to the contact positions. With (a) an emission signal at an arbitrary frequency $60 \mathrm{kHz}$; or (b) at a resonance frequency $79.620 \mathrm{kHz}$.

point, except at boundaries. The neighborhood consists of eight neighboring points surrounding the point of interest

$$
d_{i, j}(f)=\sum_{m=i-1}^{i+1} \sum_{n=j-1}^{j+1}\left|R_{m, n}(f)-R_{i, j}(f)\right| /\left[8 * R_{i, j}(f)\right] .
$$

Then a mean derivative contrast value $C(f)$ for the entire ADDP image is calculated for one frequency which yields

$$
C(f)=\sum_{i=2}^{69} \sum_{j=2}^{59} d_{i, j}(f) /\left(58^{*} 68\right) .
$$

Applied to Fig. 3, the contrast values are 0.0134 at $60 \mathrm{kHz}$ and 0.0247 at $79.62 \mathrm{kHz}$. This indicates that the ADDP is more spatially contrasted at resonance frequency. Uneven ADDP is not suitable for several reasons.

First, ADDP at resonance frequencies depends a lot on boundary conditions of the object and also changes with ambient temperature variations. This would make it difficult to ensure process reliability even if frequency shifts due to temperature variations can be calculated and compensated. ${ }^{11}$ However, it is not the case with boundary condition variations which cannot be predicted and compensated.

Second, at resonance frequencies, as ADDP is highly inhomogeneous and spatially redundant, as shown in Fig. 3(b), many areas have the same amplitude variation values. As a consequence, it becomes difficult to clearly identify the contact position among several other eligible contact points.

Intermediate $C(f)$ values are associated with more regular ADDP which allow establishing a relationship between an amplitude signal variation and a contact point.

Very small $C(f)$ values are not suitable either, because it means uniform ADDP and then an impossibility to discriminate one contact position from the other.

As a consequence, nonresonant intermediate $C(f)$ values is the best criterion to validate a particular frequency. We propose a maximum threshold value of 0.02 and a minimum threshold value of 0.01 , which have been tested and validated in our experiments with a copper plate and a liquid crystal display (LCD) screen; however, these values may be adjusted according to the situation.

Once a particular frequency is selected, we propose a criterion based on correlating a prospective ADDP to previously selected ones, to find out if adding another frequency $f^{\prime}$ helps improving the localization resolution. Following previous criterion, that frequency should first have a medium $C(f)$ value, then, its associated ADDP spatial distribution should be significantly different from all previous ones, because otherwise it would give similar information as regards discrimination of the contact point. Consequently, if there are already $N$ frequency components in the excitation signal, the 

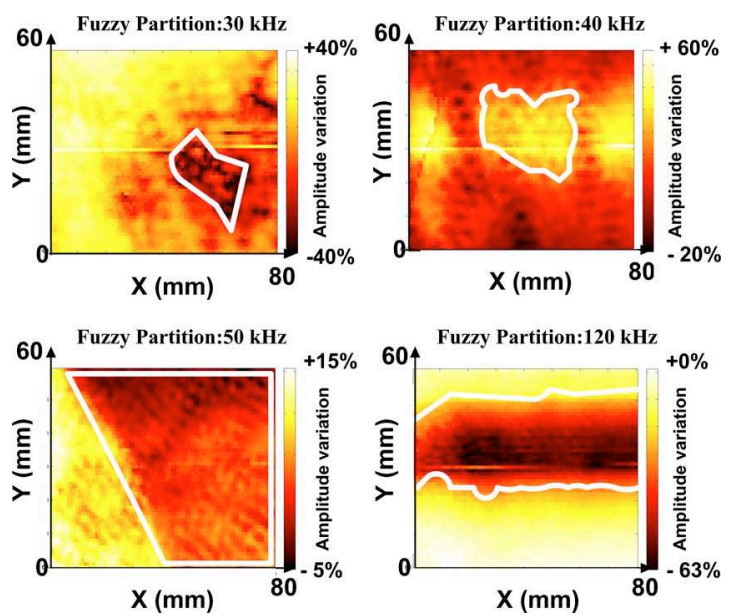

FIG. 4. (Color online) Fuzzy partitions at different frequencies. Large number of diversified partitions makes it possible to find out a precise position of contact.

choice of adding a frequency component will depend on the value of a formula expressing the global correlation value between one prospective ADDP to all the others.

The correlation function between two ADDP is expressed by $\operatorname{Corr}\left[\operatorname{ADDP}\left(f_{1}\right)\right.$ and $\left.\operatorname{ADDP}\left(f_{2}\right)\right]$. Then global non correlation value (GNC) is expressed by the formula

$$
\left\{\begin{array}{l}
\operatorname{GNC}(0)=1, \\
\operatorname{GNC}(N+1)=\operatorname{GNC}(N)^{*} 2^{N *} \prod_{i=1}^{N}\left[1-\operatorname{Corr}\left(f_{i}, f^{\prime}\right)\right] .
\end{array}\right\}
$$

Where $\operatorname{GNC}(N+1)$ is the revised value of GNC after adding a frequency. An additional frequency is accepted if the revised GNC value stays above 0.5 .

Amplitude variations of acoustic signals are analyzed to make fuzzy partitions of the tactile surface and to indicate which part of the object is touched by the finger. If different frequencies have different ADDP as shown in Fig. 4, we can combine several fuzzy partitions to go back to the exact position of contact with a high spatial resolution.

This ADDP tactile method is completely different from the absorption method ${ }^{1}$ in previous work. Indeed, the absorption method is based on amplitude variations at resonance frequencies. With this method, working at resonance frequency is almost necessary because amplitudes of signal variation are easier to measure with thick materials. In this work we use only nonresonant frequencies as they have lower contrast values $C(f)$.

Our tactile process has been tested with different objects, such as glass plates, curved copper shells, or the upper layer of LCD screen. Figure 5 (video) illustrates the process applied to a 4 in. LCD screen. We use two emitters and one PZT receiver, all arbitrary shaped. $13 \times 10$ predefined tactile points are calibrated in the training step, a matrix of reference signals is then obtained.

The acoustic wave emitters and the receiver are placed on different layers, thus the acoustic waves propagate through the whole solid before being measured. This touch screen has a response time lower than $10 \mathrm{~ms}$. As a mean of

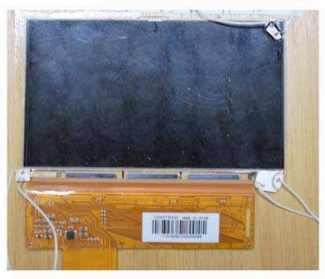

Mounting Elements

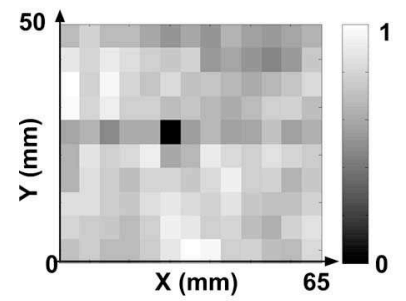

Contrast Image based on Normalized Distances

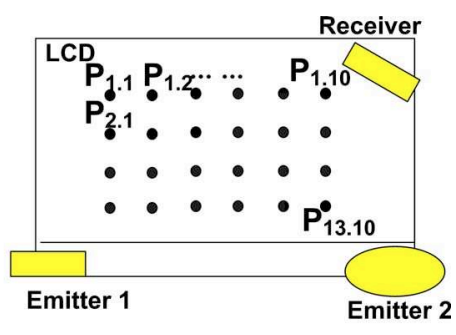

Training Process

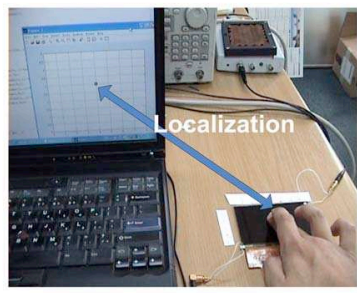

Human Computer Interaction
FIG. 5. (Color online) Application of the disturbed diffraction pattern tactile method to a LCD screen. The map of normalized distances shows clear distinction between adjacent points (enhanced online) [URL: http://dx.doi.org/10.1063/1.3276549.1].

comparison, a time reversal process requires as many boundary reflections as possible to increase reliability and then a longer time window in order to digitize and cross-correlate acoustic signals. It is therefore more time consuming. With the presented ADDP method, a $4 \mathrm{~ms}$ acquisition window is enough.

In this letter, a tactile acoustic process based on ADDP is proposed and tested to transform various solids such as metal plates or LCD screens to real-time, drag-and-drop capable interactive objects. A method to select the frequency of the excitation signals is suggested. It is based on the calculation of normalized distances and selects preferably nonresonant frequencies. It requires simple signal processing and its efficiency in various solids has been proved. As it is derived from a time reversal approach, both methods share almost the same advantages and the same physical limitations. However, this active method allows the use of simple finger touch, which constitutes a real advance compared to previous work in state of the art.

${ }^{1}$ R. K. Ing, N. Quieffin, S. Catheline, and M. Fink, Appl. Phys. Lett. 87, 204104 (2005).

${ }^{2}$ R. K. Ing, N. Quieffin, S. Catheline, and M. Fink, J. Acoust. Soc. Am. 117, 2560 (2005).

${ }^{3}$ R. Adler and P. Desmares, IEEE Trans. Ultrason. Ferroelectr. Freq. Control 34, 195 (1987).

${ }^{4}$ R. Johnson, U.S. Patent No. 3,673,327 (1972).

${ }^{5}$ J. P. Nikolovski and D. Royer, Proc.-IEEE Ultrason. Symp. 1, 699 (1997).

${ }^{6}$ E. Dieulesaint, D. Royer, and O. Legras, French Patent No. FR8903074, Publication Nb: 2644309 (1989).

${ }^{7}$ K. Nicol and E. M. C. Hennig, U.S. Patent No. 4,134,063 (1979).

${ }^{8} \mathrm{~S}$. Malik and J. Laszlo, Proceedings of the 6th International Conference on Multimodal Interfaces (State College, PA, USA, October 13-15, 2004), Vol. 1, p 289.

${ }^{9}$ W. Westerman and J. G. Elias, U.S. Patent No. 6,323,846 (2001).

${ }^{10}$ R. K. Ing, J. P. Nikolovski, D. Cassereau, and M. Fink, J. Acoust. Soc. Am. 123, 3643 (2008).

${ }^{11}$ G. Ribay, S. Catheline, D. Clorennec, R. K. Ing, N. Quieffin, and M. Fink, IEEE Trans. Ultrason. Ferroelectr. Freq. Control 54, 378 (2007). 